\title{
Cross-linked Polymersomes with Reversible Deformability and Oxygen Transportability
}

Jiwon Kim, ${ }^{1}$ Sungwoo Jeong, ${ }^{2}$ Roman Korneev, ${ }^{3}$ Kwanwoo Shin,,${ }^{2, *}$ and Kyoung Taek Kim ${ }^{1, *}$

${ }^{1}$ Department of Chemistry, Seoul National University, Seoul 08826, Korea

${ }^{2}$ Department of Chemistry and Institute of Biological Interfaces, Sogang University, Seoul 04107, Korea

${ }^{3}$ Center for Hybrid Nanostructures, University of Hamburg, Hamburg 22607, Germany

*Correspondence to:

(K.T.K.) E-mail ktkim72@snu.ac.kr

(K.S.) E-mail kwshin@sogang.ac.kr

\section{CONTENTS}

1. Synthesis of hydrophilic modules

2. Synthesis of hydrophobic modules

3. Modular synthesis of block copolymers

4. Solution self-assembly and cross-linking

5. Micropipette aspiration experiments

6. UV-vis spectroscopy for ZnTPP loaded vesicles

7. Hemoglobin-encapsulating polymersomes

8. References 
1. Synthesis of hydrophilic modules<smiles>COCCC(C)(C)COC</smiles>

29
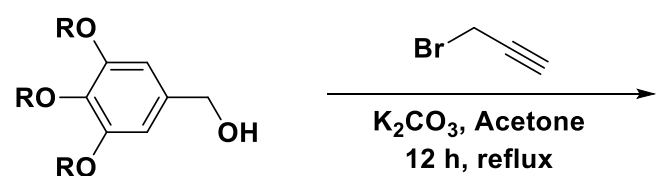

$12 \mathrm{~h}$, reflux

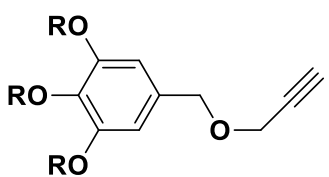

$\mathrm{R}=\mathrm{CH}_{3}\left(\mathrm{OCH}_{2} \mathrm{CH}_{2}\right)_{12}$

Scheme S2. Synthesis of PEG5503-propargyl ether

PEG5503-4-propargyl ether. A mixture of PEG5503-OH (4 g, $2.22 \mathrm{mmol})$ and propargyl bromide $(0.57 \mathrm{~mL}, 5.11 \mathrm{mmol}, 2.3 \mathrm{eq} ., 80 \mathrm{wt} \%$ in toluene) was stirred for $10 \mathrm{~min}$ in acetone $(100 \mathrm{~mL}) . \mathrm{K}_{2} \mathrm{CO}_{3}(5 \mathrm{~g})$ was then added, and the reaction was stirred for $18 \mathrm{~h}$ under reflux. Thereafter, the solid was removed by filtration and acetone was evaporated. Water was added and the mixture was extracted with dichloromethane. The organic phase was dried over $\mathrm{MgSO}_{4}$ and filtered. The solvent was removed by rotary evaporation and then mixture was purified by flash column chromatography using silica gel (dichloromethane : methanol = 95:5 v/v). The compound was obtained as yellow solid. Yield: $4.01 \mathrm{~g}, 93 \%$. ${ }^{\mathbf{1}} \mathbf{H}$ NMR (400 MHz, 
$\left.52 \mathrm{CDCl}_{3}\right) 6.59(\mathrm{~s}, 2 \mathrm{H}), 4.49(\mathrm{~d}, J=5.2 \mathrm{~Hz}, 2 \mathrm{H}), 4.18(\mathrm{t}, J=5.2 \mathrm{~Hz}, 4 \mathrm{H}), 4.14(\mathrm{t}, J=5.2 \mathrm{~Hz}, 2 \mathrm{H})$,

53 3.93-3.45 (m, - $\left.\mathrm{CH}_{2} \mathrm{CH}_{2} \mathrm{O}-\right), 3.39(\mathrm{~s}, 9 \mathrm{H}), 2.49(\mathrm{t}, J=5.6 \mathrm{~Hz}, 1 \mathrm{H}) \mathrm{ppm} ;{ }^{13} \mathbf{C} \mathbf{N M R}(400 \mathrm{MHz}$,

$\left.54 \mathrm{CDCl}_{3}\right)$ 152.60, 137.49,137.22, 106.50, 72.29, 71.95, 70.77-70.57, 69.83, 68.79, 64.86, 59.9,

$55 \quad 59.07,78.7,76.4 \mathrm{ppm}$.

56

57

\section{Synthesis of hydrophobic modules}

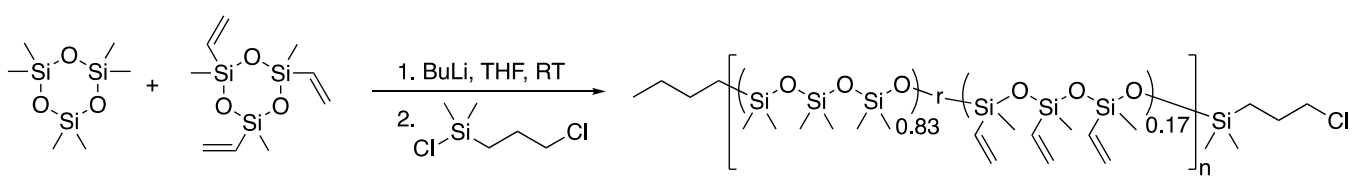

$$
\begin{aligned}
& \mathrm{NaN}_{3}, \mathrm{TBAB}, \mathrm{THF}
\end{aligned}
$$

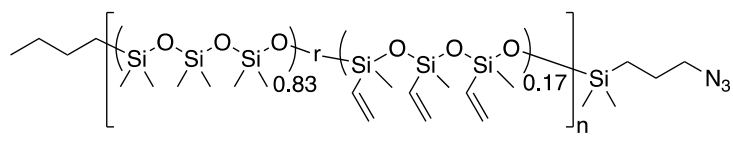

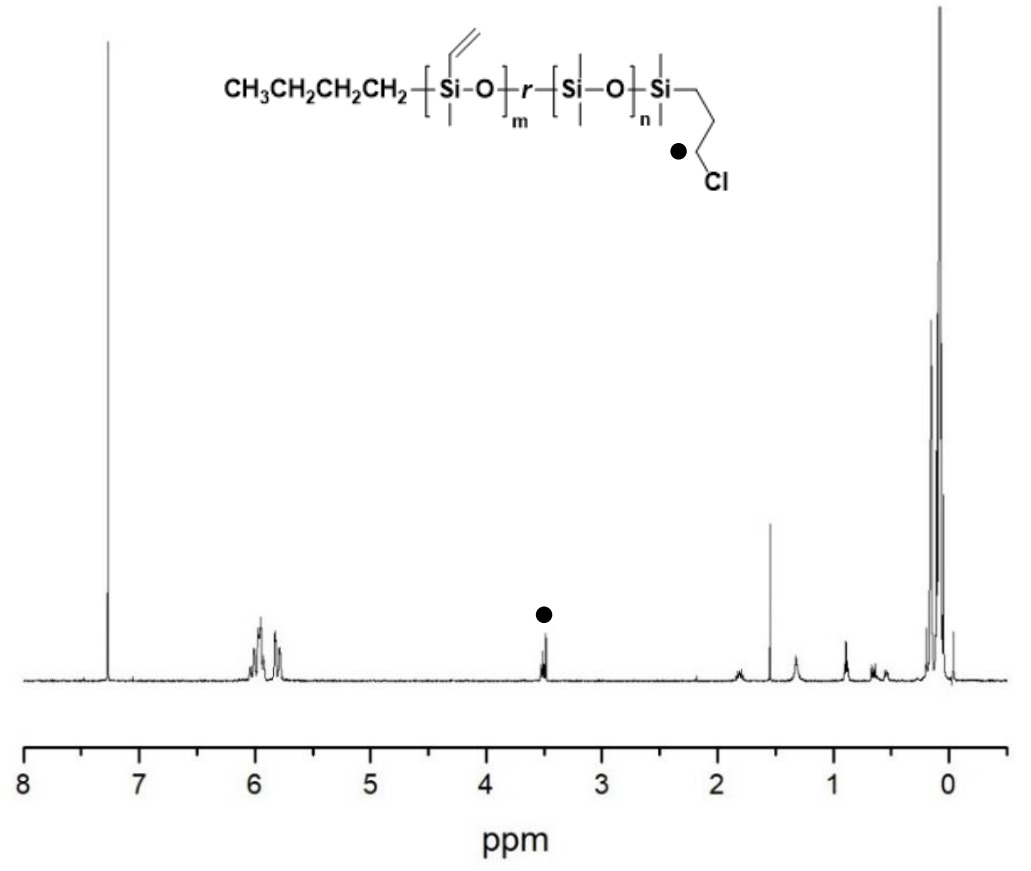

62

Figure S1. ${ }^{1} \mathrm{H}$ NMR $\left(500 \mathrm{MHz}, \mathrm{CDCl}_{3}\right)$ spectrum of $\omega$-chloro-PDMVS 


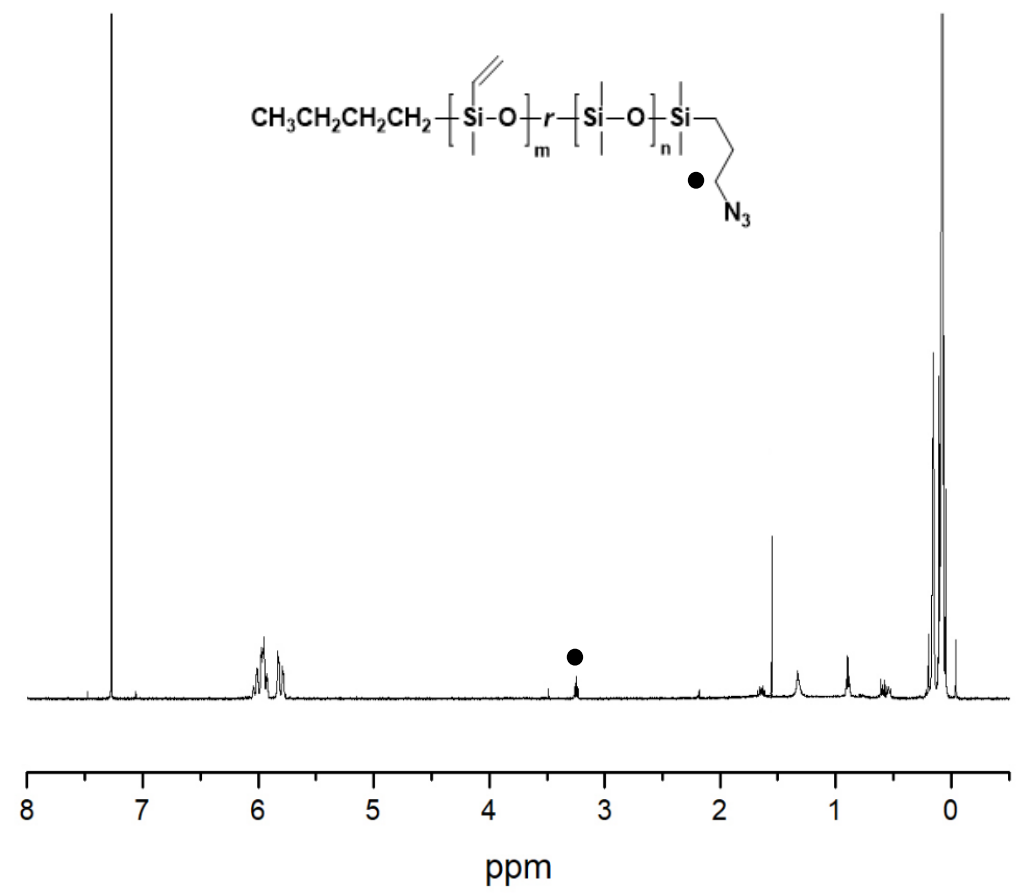

64

65

66

67

68

3. Modular synthesis of block copolymers
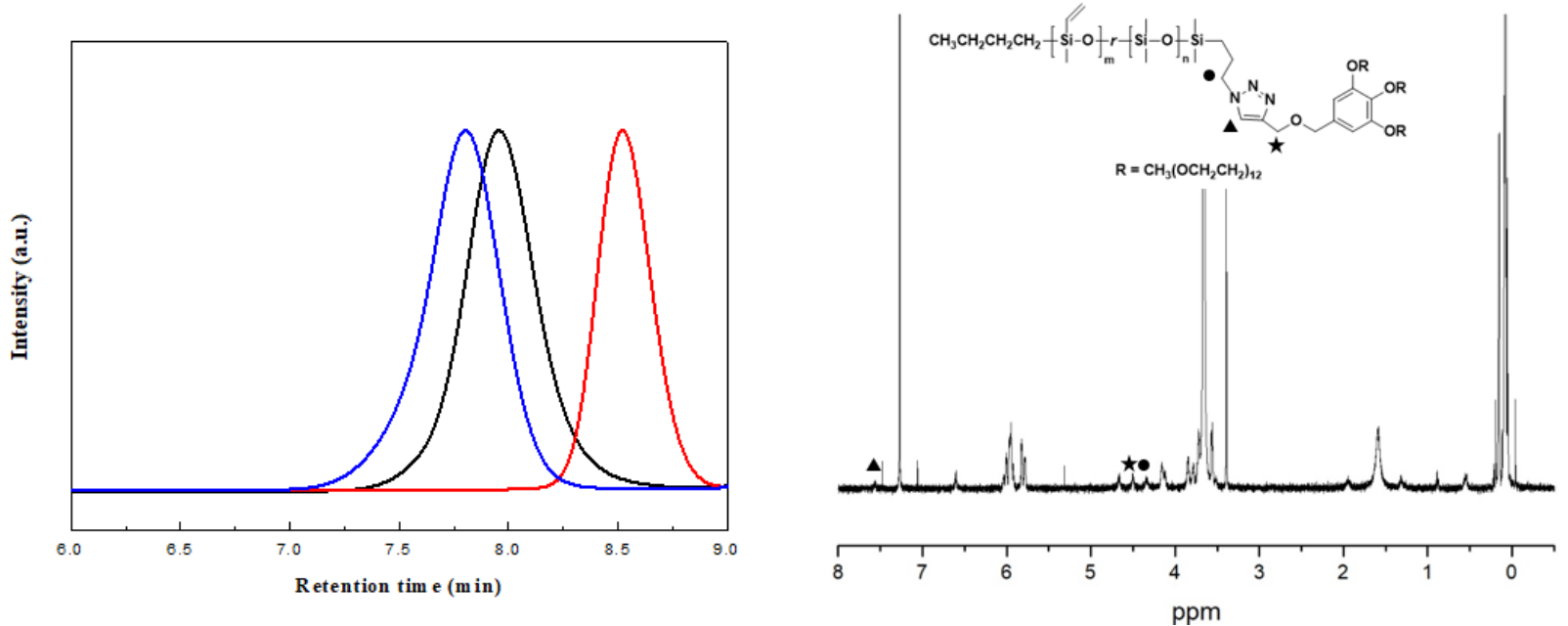

69

Figure S3. GPC analysis (A) and ${ }^{1} \mathrm{H}$ NMR spectrum (B) of PDMVS- $b$-PEG550 3 


\section{Self-assembly and cross-linking}

Solution self-assembly. These BCPs were allowed to self-assemble from a dioxane solution

$(2 \mathrm{~mL}, 1 \mathrm{wt} \%)$ by adding an equal volume of water at a controlled rate $\left(1 \mathrm{~mL} \mathrm{~h}^{-1}\right)$ with stirring. To the solution (dioxane : water = $1: 1$ for volume ratio), $5 \mathrm{mg}$ of 2-Hydroxy-4'-(2hydroxyethoxy)-2-methylpropiophenone (Irgacure 2959) was added for cross-linking. The solution was stirred for $5 \mathrm{~h}$ under UV lamp $(\lambda=365 \mathrm{~nm})$, followed by dialysis against water. All BCPs were self-assembled under identical conditions for comparison.

Direct hydration. These BCPs were dispersed to $500 \mu \mathrm{L}$ of water. After $3 \mathrm{~h}, 100 \mu \mathrm{L}$ of the solution was diluted with water (x 10). To the solution, 2-Hydroxy-4'-(2-hydroxyethoxy)-2methylpropiophenone (Irgacure 2959) (5 mg) was added for cross-linking. The solution was stirred for $5 \mathrm{~h}$ under UV lamp $(\lambda=365 \mathrm{~nm})$, followed by dialysis against water. All BCPs were self-assembled under identical conditions for comparison.
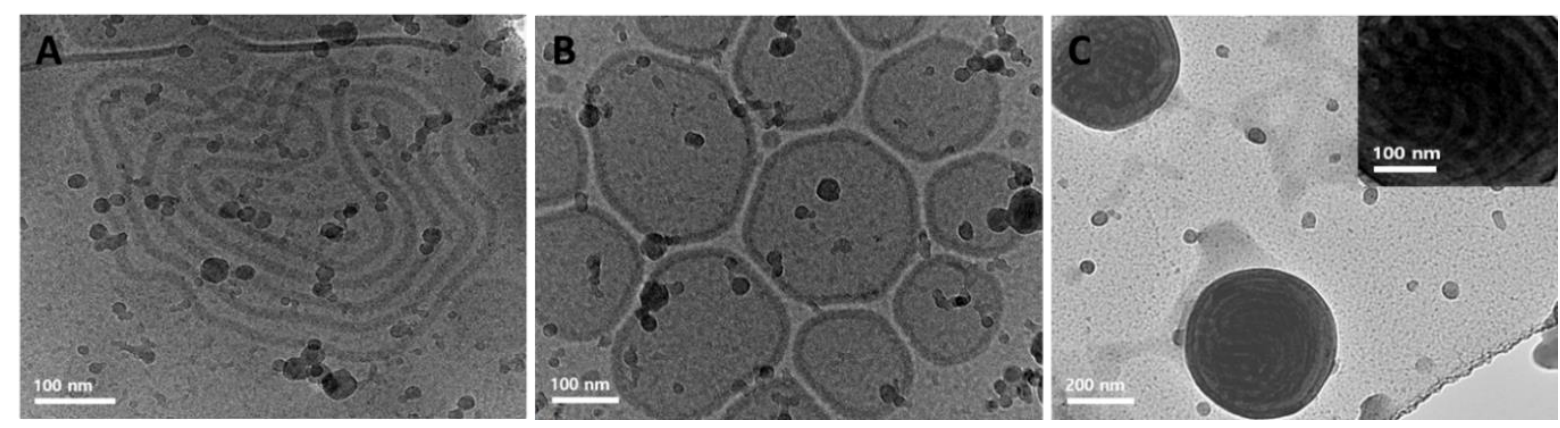

Figure S4. Uncross-linked morphological transition upon reduction of the chain length of PDMVS. Cryo-EM images of (A) cylindrical micelles (PEG5503-PDMVS15K), (B) polymersomes (PEG5503 - PDMVS20K), and (C) hexagonal nanoparticles (PEG5503-PDMVS $26 \mathrm{~K})$.

Difference of observed morphology of self-assembled structures. The BCPs with PDMVS having different molecular weights (PEG5503-PDMVS15K and PEG5503-PDMVS20K) formed polymersomes under different conditions. PEG5503-PDMVS20K only formed polymersomes by the co-solvent method (dioxane/water). However, PEG5503 $-\mathrm{PDMVS15K}$ could directly self-assembled into polymersomes in water. We used PEG5503-PDMVS20K for 
103

the morphological and physical characterization of the polymersomes because of their superior stability. In case of protein encapsulation, we used PEG5503-PDMVS15K for preventing denaturation of hemoglobin. While the PEG5503-PDMVS26K formed hexosomes, or nanoparticles composed of the inverse-hexagonal phase via solution self-assembly (Figure $\mathrm{S} 4 \mathrm{C}$ ), the BCP could not be directly dispersed in water due to its high-MW hydrophobic block.

\section{Table S1. Difference of observed morphology of self-assembled structures}

$\begin{array}{cccc}\text { Entry } & \text { BCPs } & \text { Solution self-assembly } & \text { Direct hydration } \\ \mathbf{1} & \text { PEG5503-PDMVS10K } & \text { Spherical micelles } & \text { Cylindrical micelles } \\ \mathbf{2} & \text { PEG550 }_{3} \text {-PDMVS15K } & \text { Cylindrical micelles } & \text { Polymersomes } \\ \mathbf{3} & \text { PEG550 } & \text { Nanoparticles } \\ \mathbf{4} & \text { PEG550 } & \text { Polymersomes } & \text { No dispersion }\end{array}$

Cross-linking procedure. Homopolymer, PDMVS $\left(M_{\mathrm{n}}=14500 \mathrm{~g} \mathrm{~mol}^{-1}\right)$ was cross-linked by using 2-Hydroxy-4'-(2-hydroxyethoxy)-2-methylpropiophenone (Irgacure 2959, sigma) as a photoradical generator. Into $4 \mathrm{~mL}$ of co-solvent (dioxane/water), $100 \mathrm{mg}$ of homopolymer and $10 \mathrm{mg}$ of Irgacure 2959 were added. Under the illumination of UV $(\lambda=365 \mathrm{~nm}, 8 \mathrm{~W})$ for $5 \mathrm{~h}$, the resulting polymer solution (about $0.02 \mathrm{~g} \mathrm{~mL}^{-1}$ ) yielded soft gel. The cross-linking of vinyl group in PMVS chain was confirmed by ${ }^{1} \mathrm{H}-\mathrm{NMR}$ spectroscopy and differential scanning calorimetry (DSC) experiments. The soluble fraction of the cross-linked network was inspected by ${ }^{1} \mathrm{H}$ NMR, which resulted in the disappearance of the characteristic peaks of pendant vinyl groups at $(\delta=5.5-6 \mathrm{ppm})$. DSC results of the cross-linked PDMVS showed a single peak $\left(T_{\mathrm{m}}\right.$ $\left.=-50.3^{\circ} \mathrm{C}\right)$ upon a heating scan, which are in contrast to the double melting peaks $\left(T_{\mathrm{m} 1}=-47.3\right.$ ${ }^{\circ} \mathrm{C}$ and $T_{\mathrm{m} 2}=-39.1{ }^{\circ} \mathrm{C}$ ) for the pristine BCP. These results indicated the formation of covalent bonds between the PMVS chains in solution in the presence of photoradical generators. ${ }^{3}$ 
A

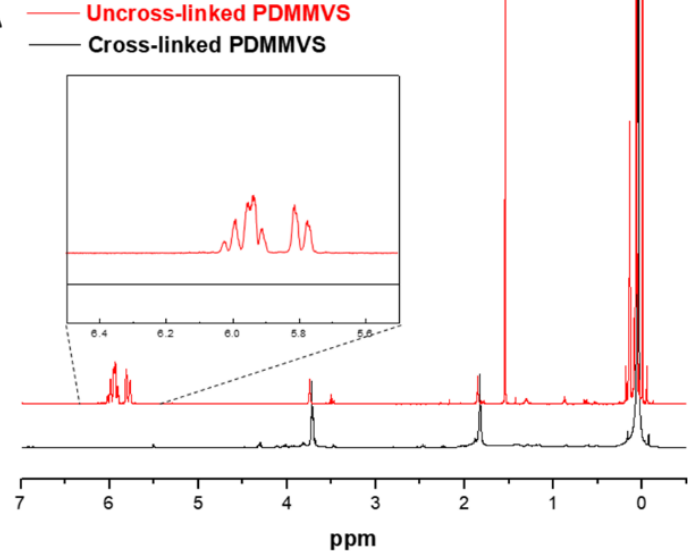

B Uncross-linked PDMMVs

Cross-linked PDMMVs
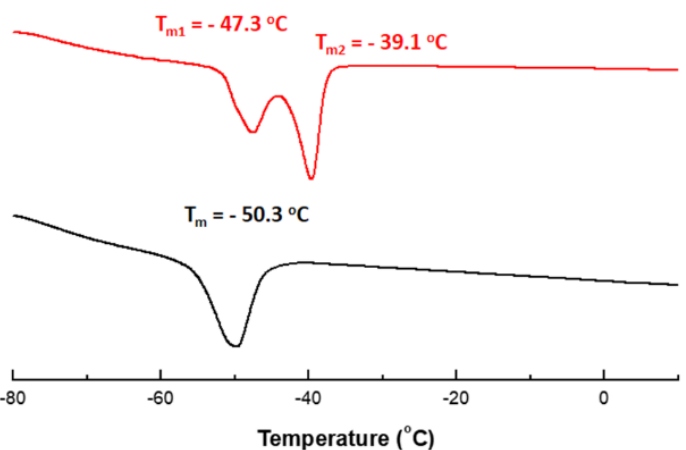

116

Figure S5. ${ }^{1} \mathrm{H}-\mathrm{NMR}$ spectroscopy and differential scanning calorimetry (DSC) analysis for cross-linking of vinyl group in PMVS chain.

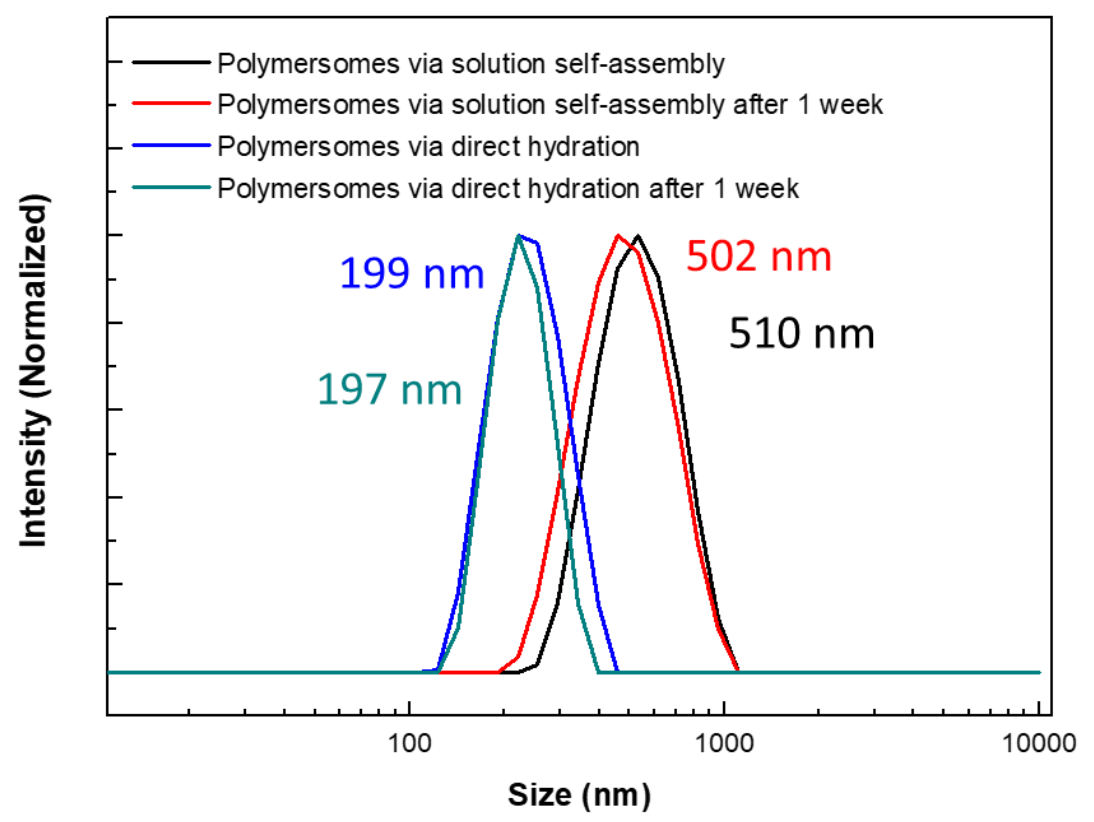

Figure S6. Dynamic light scattering (DLS) size plots of polymersomes of PEG5503-b-PDMVS self-assembled via co-solvent and direct hydration methods. The polymersomes encapsulating 


\section{Micropipette aspiration experiments}

Observation of microdeformed PEG- $b$-PDMVS polymersomes. For quantitative microdeformation experiments, the aspiration pressure $\mathrm{P}$ was controlled by adjusting the height of the oil-filled reservoir connected to the back of the pipette. Membrane tension $\sigma$ was computed from $\sigma=K_{\mathrm{a}} \alpha$ (eq 1). Here, $\sigma$ represents the membrane tension. The area strain, $\alpha$, is determined by $\mathrm{A} / \mathrm{A}_{0}$, where $\mathrm{A}_{0}$ is the initial membrane area and $\mathrm{A}$ is the change in area due to application of pressure. Image analysis was performed using the public domain software ImageJ. A semi-automatic tracking procedure was implemented to monitor the tongue length position in time. Pipette and vesicle radii were measured separately for each set of experiments.

Capacity for polymersomes to undergo deflation-inflation cycles. The cross-linked polymersomes underwent the deflation-inflation cycle without losing structural integrity. In contrast, the unlinked polymersomes did not retain their structures under the same stresses. In other words, unlinked polymersomes failed to retain their structures by negative pressure into polymersomes.
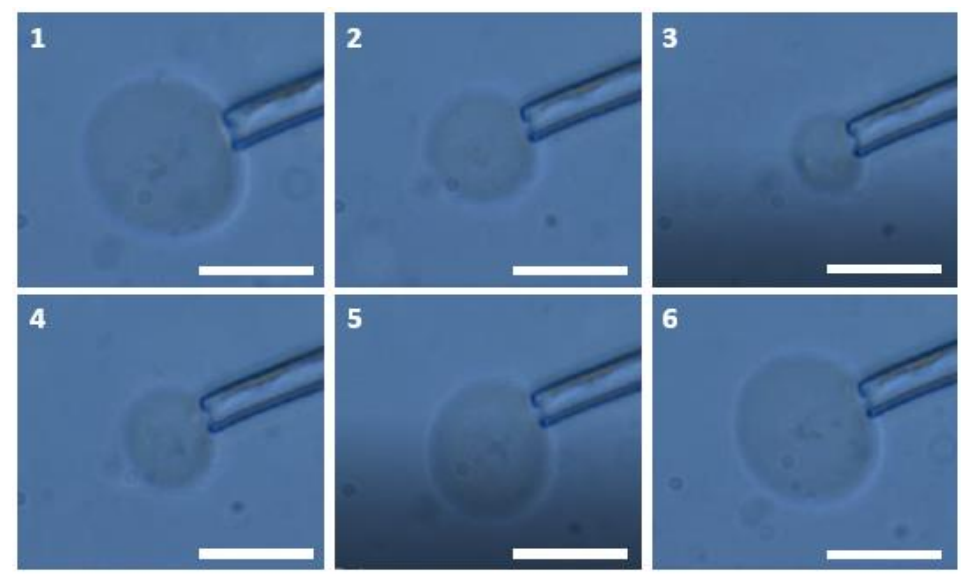

Figure S7. Sequence of a locally cross-linked vesicle of PEG- $b$-PDMVS. The crosslinked vesicle was partially deflated ( 1 to 3 ) by a negative pressure and inflation under positive pressure brought the vesicle back to its original shape (4 to 6 ) (Scale bar $=20$ $\mu \mathrm{m})$. 


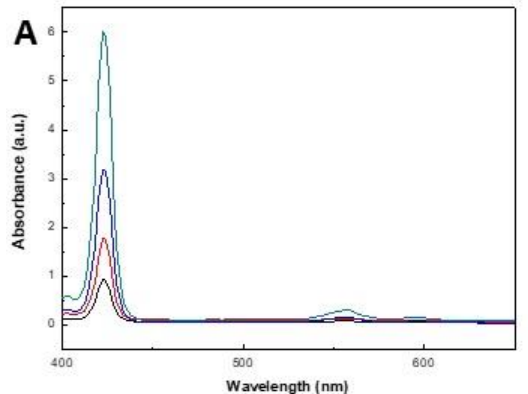
pressure (1 to 3$)($ Scale bar $=20 \mu \mathrm{m})$.

\section{ZnTPP loaded vesicles} the polymer mass.
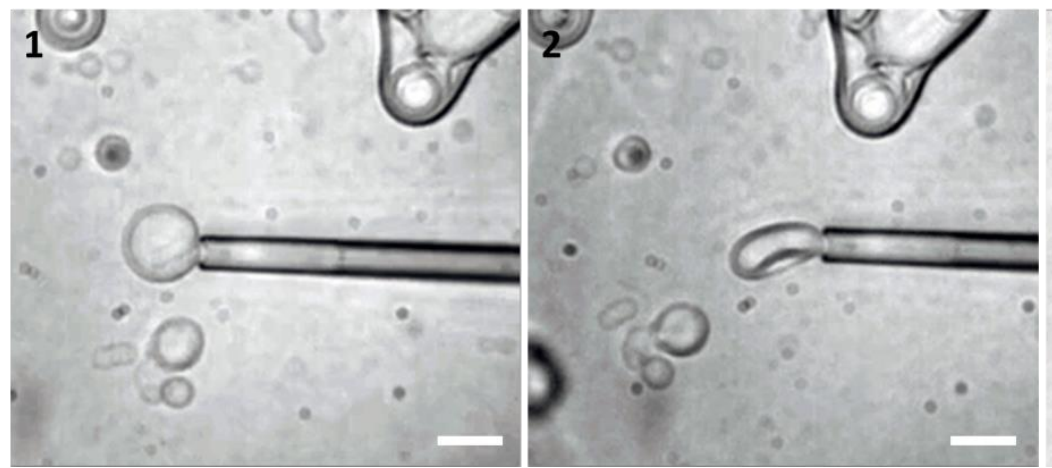

\section{3}

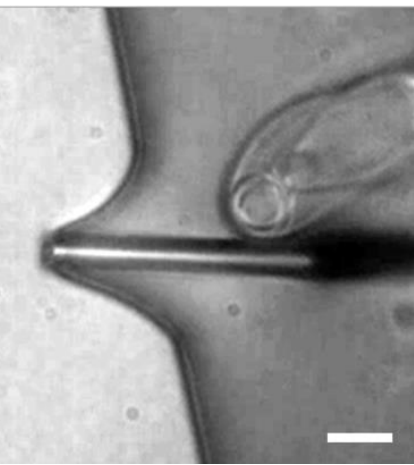

Figure S8. Sequence of an uncross-linked vesicle of PEG- $b$-PDMVS. The uncross-linked polymersomes failed to retain their round-shape and then finally were ruptured under negative

\section{Plot of calibration curve to determine ZnTPP loading amount ${ }^{3}$}

The ZnTPP loading amount within aqueous vesicles was determined by comparison of its vesicle absorption at $424 \mathrm{~nm}$ with the calibration of free ZnTPP. To $1 \mathrm{~mL}$ of ZnTPP loaded vesicles dispersion, addition of $1 \mathrm{~mL}$ of THF allowed to eliminate the effect of nanoparticle-induced scatter on the observed absorption by ZnTPP. According to the calibration curve, the ZnTPP loading amount was approximately $0.19 \mathrm{wt} \%$ relative to

Figure S9. (A) Absorptions of free ZnTPP with different amounts (B) Plotting of a calibration curve of free ZnTPP, and (C) Absorption of ZnTPP loaded vesicles after elimination of the effect of nanoparticle-induced scattering. 


\section{Hemoglobin-Encapsulating Polymersomes}

To prevent hemoglobin $(\mathrm{Hb})$ from oxidizing to methemoglobin (metHb) during the preparation of hemoglobin-encapsulating polymersomes (HEPs), $\mathrm{Hb}$ was complexed with carbon monoxide (CO) before encapsulation. The bovine $\mathrm{Hb}$ was diluted with phosphate-buffered saline (PBS, pH 7.3) to $30 \mathrm{mg} / \mathrm{mL}$. A few drops of sodium dithionite was added to the stock solution to form deoxyhemoglobin (deoxyHb) and then stabilized under a $\mathrm{CO}$ atmosphere to form deoxyhemoglobin and then stabilized under a $\mathrm{CO}$ atmosphere to generate CO-bound hemoglobin form (carboxyhemoglobin or $\mathrm{COHb}$ ). The prepared carboxyhemoglobin stock solution was saturated with different gases were confirmed with UV-vis spectroscopic analysis.

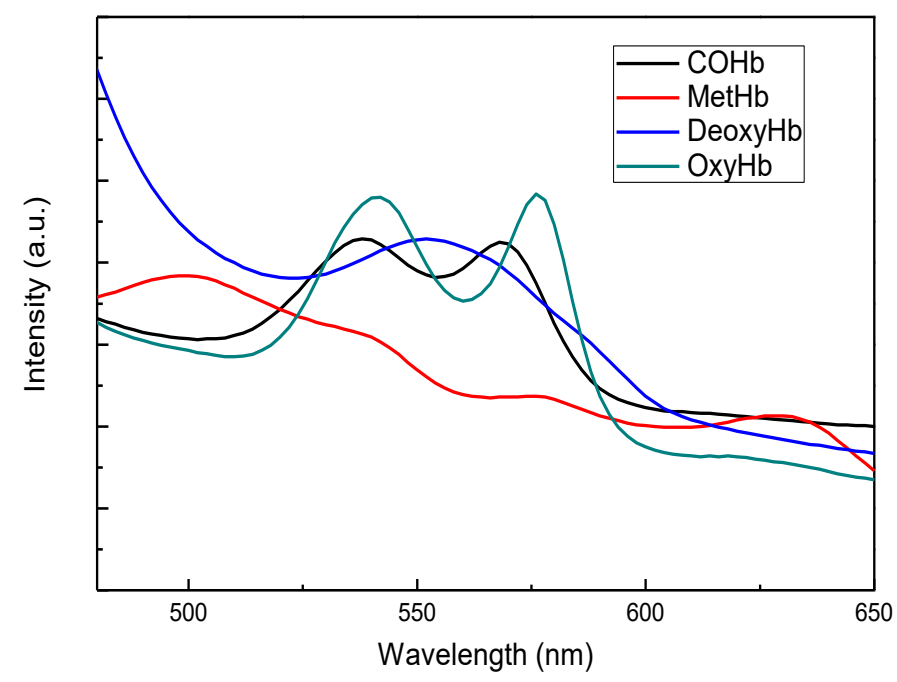

Figure S10. UV-vis spectra of $\mathrm{COHb}$, metHb, deoxyHb, and oxyHb. The commercially available bovine hemoglobin was reduced by Sodium dithionite, followed by saturation with $\mathrm{CO}$ gas to obtain $\mathrm{COHb}$.

\section{Preparation of Hemoglobin-encapsulating polymersome (HEP)}

$10 \mathrm{mg}$ of PEG5503-b-PDMVS15K was coated in $50 \mathrm{~mL}$ of a round bottom flask as a thin film. $500 \mu \mathrm{L}$ of the prepared $\mathrm{COHb}$ stock solution was added for dispersion at $4^{\circ} \mathrm{C}$. After $16 \mathrm{~h}$, any other aggregated particles were also removed by pipetting. The giant polymersomes were extruded through $200 \mathrm{~nm}$ polycarbonate membrane filter following $\mathrm{Hb}$ encapsulation to adjust their diameter to $200 \mathrm{~nm}$. Unencapsulated $\mathrm{Hb}$ was removed from the aqueous suspension by 
dialysis against PBS (a $100 \mathrm{~K}$ cut off). After cross-linking of the polymersomes with Irgacure 2959 under UV light (365 nm).

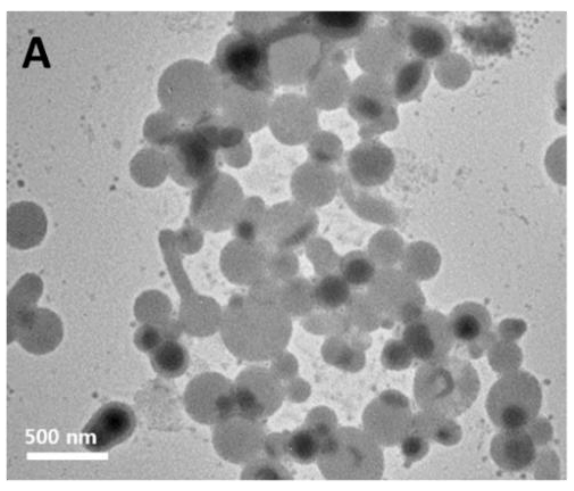

Figure S11. TEM image and DLS size plot for size-controlled polymersomes containing hemoglobin for oxygen transportation.

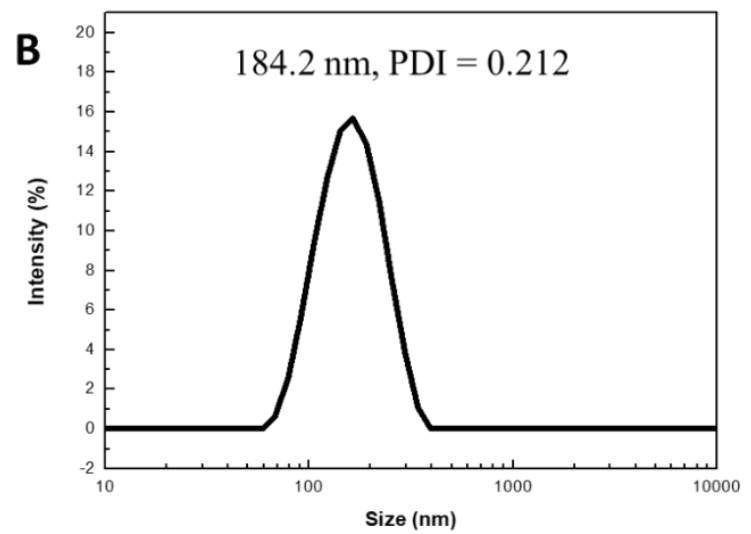

\section{Plot of calibration curve to determine Hb encapsulation efficiency (EE\%)}

We estimated the EE\% of $\mathrm{Hb}$ by measuring the absorption of the dispersion after purification. For encapsulation, the carboxyhemoglobin $(\mathrm{COHb}, 30 \mathrm{mg} / \mathrm{mL})$ in PBS was used for direct hydration of thin films of PEG5503-PDMVS15K. After hydration, the resulting dispersion was purified by dialysis against PBS, followed by size exclusion chromatography (sephadex G). The purified polymersome solution was diluted 30 times with PBS. The absorption of the dispersion was measured by UV-Vis., which was compared to the calibration plot obtained using $\mathrm{COHb}$ in PBS. The estimated EE\% in our case was $8.37 \%$.
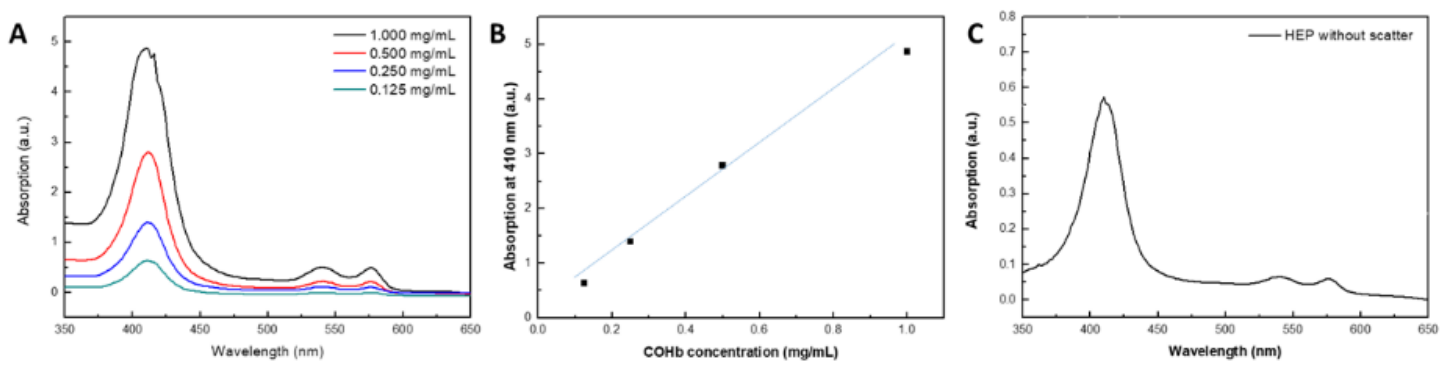

Figure S12. (A) Absorptions of free COHb with different amounts (B) Plotting of suitable calibration curve of free $\mathrm{COHb}$, and (C) Absorption of hemoglobin loaded vesicles after elimination of the effect of nanoparticle-induced scattering. 


\section{Oxygen-nitrogen cycling experiments with HEP}

213 To investigate the oxygen binding of HEP, UV-vis spectrometry was used. The solution 214 containing HEP was sealed off from the surrounding air and deoxygenated by repeated 215 evacuation and flushing with pure $\mathrm{N}_{2}$ for $150 \mathrm{~min} .2 \mathrm{~mL}$ of suspension was analyzed by UV216 vis spectrometry to observe the characteristic peaks of deoxyHb. Introducing oxygen to the 217 suspension was performed by pure $\mathrm{O}_{2}$ saturation for $60 \mathrm{~min}$. The oxygen-nitrogen exchange 218 process was repeated at least three times.

\section{References}

(1) Jeong, M. G.; van Hest, J. C. M.; Kim, K. T. Self-assembly of dendritic-linear block copolymers with fixed molecular weight and block ratio. Chem. Comm. 2012, 48, 3590-3592.

(2) La, Y.; Park, C.; Shin, T. J.; Joo, S. H.; Kang, S.; Kim, K. T. Colloidal inverse bicontinuous cubic membranes of block copolymers with tunable surface functional groups. Nat. Chem. 2014, 6, 534-541.

(3) Dollase, T.; Spiess, H. W.; Gottlieb, M.; Yerushalmi-Rozen, R. Crystallization of PDMS: The effect of physical and chemical crosslinks. Europhys. Lett. 2002, 60, 390-396.

(4) Yeow, J.; Shanmugam, S.; Corrigan, N.; Kuchel, R. P.; Xu, J.; Boyer, C. A Polymerization230 Induced Self-Assembly Approach to Nanoparticles Loaded with Singlet Oxygen Generators. Macromolecules 2016, 49, 7277-7285 